\title{
Generalized Hermite -Hadamard type integral inequalities for functions whose 3rd derivatives are s-convex
}

\author{
Mehmet Zeki Sarikaya and Hüseyin Budak \\ Department of Mathematics, Faculty of Science and Arts, Düzce University, Düzce-Turkey \\ E-mail: sarikayamz@gmail.com, hsyn.budak@gmail.com
}

\begin{abstract}
In this paper, we have established Hermite-Hadamard type inequalities for functions whose 3rd derivatives are s-convex depending on a parameter. These results have generalized some relationships with [4].
\end{abstract}

2010 Mathematics Subject Classification. 26D07. 26D10, 26D15, 26A33

Keywords. Hermite-Hadamard's inequalities, $s$-convex function, Hölder's inequality.

\section{Introduction}

Definition 1.1. The function $f:[a, b] \subset \mathbb{R} \rightarrow \mathbb{R}$, is said to be convex if the following inequality holds

$$
f(\lambda x+(1-\lambda) y) \leq \lambda f(x)+(1-\lambda) f(y)
$$

for all $x, y \in[a, b]$ and $\lambda \in[0,1]$. We say that $f$ is concave if $(-f)$ is convex.

The inequalities discovered by C. Hermite and J. Hadamard for convex functions are very important in the literature (see, e.g., [6],[10, p.137]). These inequalities state that if $f: I \rightarrow \mathbb{R}$ is a convex function on the interval $I$ of real numbers and $a, b \in I$ with $a<b$, then

$$
f\left(\frac{a+b}{2}\right) \leq \frac{1}{b-a} \int_{a}^{b} f(x) d x \leq \frac{f(a)+f(b)}{2} .
$$

We note that Hadamard's inequality may be regarded as a refinement of the concept of convexity and it follows easily from Jensen's inequality. Hadamard's inequality for convex functions has received renewed attention in recent years and a remarkable variety of refinements and generalizations have been found (see, for example, $[1,2,6,7,10]$ ) and the references cited therein.

Definition 1.2. [3] Let $s$ be a real numbers, $s \in(0,1]$. A function $f:[0, \infty) \rightarrow[0, \infty)$ is said to be $s$-convex (in the second sense), or that $f$ belongs to the class $K_{s}^{2}$, if $f$

$$
f(\alpha x+(1-\alpha) y) \leq \alpha^{s} f(x)+(1-\alpha)^{s} f(y)
$$

for all $x, y \in[0, \infty)$ and $\alpha \in[0,1]$.

An $s$-convex function was introduced in Breckner's paper [3] and a number of properties and connections with $s$-convexity in the first sense are discussed in paper [8]. Of course, $s$-convexity means just convexity when $s=1$.

Tbilisi Mathematical Journal 7(2) (2014), pp. 41-49.

Tbilisi Centre for Mathematical Sciences.

Received by the editors: 17 June 2014

Accepted for publication: 12 November 2014 
Lemma 1.3. Let $f: I \subseteq \mathbb{R} \rightarrow \mathbb{R}$ be a three times differentiable function on $I^{\circ}$ with $a, b \in I$ and $a<b$. If $f^{\prime \prime \prime} \in L[a, b]$, then

$$
\begin{aligned}
& \frac{f(a)+f(b)}{2}-\frac{1}{b-a} \int_{a}^{b} f(x) d x-\frac{b-a}{12}\left[f^{\prime}(b)-f^{\prime}(a)\right] \\
= & \frac{(b-a)^{3}}{12} \int_{0}^{1} t(1-t)(2 t-1) f^{\prime \prime \prime}[t b+(1-t) a] d t .
\end{aligned}
$$

In [4], Chun and Qi establish the following inequalities:

Theorem 1.4. Let $f:[a, b] \rightarrow \mathbb{R}$ be a three times differentiable mapping on $(a, b)$ with $0 \leq a<b$. If $\left|f^{\prime \prime \prime}\right|^{q}$ is $s$-convex on $[a, b]$ for same fixed $s \in(0,1]$ and $q \geq 1$, then

$$
\begin{aligned}
& \left|\frac{f(a)+f(b)}{2}-\frac{1}{b-a} \int_{a}^{b} f(x) d x-\frac{b-a}{12}\left[f^{\prime}(b)-f^{\prime}(a)\right]\right| \\
\leq & \frac{(b-a)^{3}}{192}\left(\frac{2^{2-s}\left(6+s+2^{s+2} s\right)}{(s+2)(s+3)(s+4)}\left[\left|f^{\prime \prime \prime}(a)\right|^{q}+\left|f^{\prime \prime \prime} b\right|^{q}\right]\right)^{\frac{1}{q}} .
\end{aligned}
$$

Theorem 1.5. Let $f:[a, b] \rightarrow \mathbb{R}$ be a three times differentiable mapping on $(a, b)$ with $0 \leq a<b$. If $\left|f^{\prime \prime \prime}\right|^{q}$ is $s$-convex on $[a, b]$ for same fixed $s \in(0,1]$ and $q>1$, then

$$
\begin{aligned}
& \left|\frac{f(a)+f(b)}{2}-\frac{1}{b-a} \int_{a}^{b} f(x) d x-\frac{b-a}{12}\left[f^{\prime}(b)-f^{\prime}(a)\right]\right| \\
\leq & \frac{(b-a)^{3}}{96}\left(\frac{1}{p+1}\right)^{\frac{1}{p}}\left(\frac{2^{1-s}\left(s 2^{s}+1\right)}{(s+1)(s+2)}\left[\left|f^{\prime \prime \prime}(a)\right|^{q}+\left|f^{\prime \prime \prime} b\right|^{q}\right]\right)^{\frac{1}{q}}
\end{aligned}
$$

where $\frac{1}{p}+\frac{1}{q}=1$.

Theorem 1.6. Let $f:[a, b] \rightarrow \mathbb{R}$ be a three times differentiable mapping on $(a, b)$ with $0 \leq a<b$. If $\left|f^{\prime \prime \prime}\right|^{q}$ is $s$-convex on $[a, b]$ for same fixed $s \in(0,1]$ and $q>1$, then

$$
\begin{aligned}
& \left|\frac{f(a)+f(b)}{2}-\frac{1}{b-a} \int_{a}^{b} f(x) d x-\frac{b-a}{12}\left[f^{\prime}(b)-f^{\prime}(a)\right]\right| \\
\leq & \frac{(b-a)^{3}}{24}\left(\frac{1}{(p+1)(p+3)}\right)^{\frac{1}{p}}\left(\frac{2}{(s+2)(s+3)}\left[\left|f^{\prime \prime \prime}(a)\right|^{q}+\left|f^{\prime \prime \prime} b\right|^{q}\right]\right)^{\frac{1}{q}}
\end{aligned}
$$

where $\frac{1}{p}+\frac{1}{q}=1$.

For more information and recent developments on this topic, please refer to $[4,5,9,11,12]$.

The aim of this paper is to establish generalized Hermite-Hadamard's inequalities for function whose 3rd derivatives in absolute value at certain powers are $s$-convex functions and these results have generalized some relationships with [4]. 


\section{Main Results}

We give a important identity for three times differentiable convex functions:

Lemma 2.1. Let $f:[a, b] \rightarrow \mathbb{R}$ be a three times differentiable mapping on $(a, b)$ with $a<b$. If $f^{\prime \prime \prime} \in L[a, b]$, then the following equality holds:

$$
\begin{aligned}
& \frac{(1-2 \lambda)^{2}(b-a)}{12}\left[f^{\prime}(\lambda a+(1-\lambda) b)-f^{\prime}(\lambda b+(1-\lambda) a)\right] \\
& -\frac{(1-2 \lambda)}{2}[f(\lambda a+(1-\lambda) b)+f(\lambda b+(1-\lambda) a)]+\frac{1}{b-a} \int_{\lambda b+(1-\lambda) a}^{\lambda a+(1-\lambda) b} f(x) d x \\
= & \frac{(1-2 \lambda)^{4}(b-a)^{3}}{12} \int_{0}^{1} t(1-t)(2 t-1) f^{\prime \prime \prime}[t(\lambda a+(1-\lambda) b)+(1-t)(\lambda b+(1-\lambda) a)] d t
\end{aligned}
$$

where $\lambda \in[0,1] \backslash\left\{\frac{1}{2}\right\}$.

Proof. It suffices to note that

$$
\begin{aligned}
I= & \int_{0}^{1} t(1-t)(2 t-1) f^{\prime \prime \prime}[t(\lambda a+(1-\lambda) b)+(1-t)(\lambda b+(1-\lambda) a)] d t \\
= & -2 \int_{0}^{1} t^{3} f^{\prime \prime \prime}[t(\lambda a+(1-\lambda) b)+(1-t)(\lambda b+(1-\lambda) a)] d t \\
& +3 \int_{0}^{1} t^{2} f^{\prime \prime \prime}[t(\lambda a+(1-\lambda) b)+(1-t)(\lambda b+(1-\lambda) a)] d t \\
& -\int_{0}^{1} t f^{\prime \prime \prime}[t(\lambda a+(1-\lambda) b)+(1-t)(\lambda b+(1-\lambda) a)] d t \\
= & -2 I_{1}+3 I_{2}-I_{3} .
\end{aligned}
$$

Integrating by parts

$$
\begin{aligned}
I_{1}= & \int_{0}^{1} t^{3} f^{\prime \prime \prime}[t(\lambda a+(1-\lambda) b)+(1-t)(\lambda b+(1-\lambda) a)] d t \\
= & \frac{f^{\prime \prime}(\lambda a+(1-\lambda) b)}{(1-2 \lambda)(b-a)}-\frac{3 f^{\prime}(\lambda a+(1-\lambda) b)}{(1-2 \lambda)^{2}(b-a)^{2}} \\
& +\frac{6 f(\lambda a+(1-\lambda) b)}{(1-2 \lambda)^{3}(b-a)^{3}}-\frac{6}{(1-2 \lambda)^{4}(b-a)^{4}} \int_{\lambda b+(1-\lambda) a}^{\lambda a+(1-\lambda) b} f(x) d x
\end{aligned}
$$


similarly,

$$
\begin{aligned}
I_{2}= & \int_{0}^{1} t^{2} f^{\prime \prime \prime}[t(\lambda a+(1-\lambda) b)+(1-t)(\lambda b+(1-\lambda) a)] d t \\
= & \frac{f^{\prime \prime}(\lambda a+(1-\lambda) b)}{(1-2 \lambda)(b-a)}-\frac{2 f^{\prime}(\lambda a+(1-\lambda) b)}{(1-2 \lambda)^{2}(b-a)^{2}} \\
& +\frac{2 f(\lambda a+(1-\lambda) b)}{(1-2 \lambda)^{3}(b-a)^{3}}-\frac{2 f(\lambda b+(1-\lambda) a)}{(1-2 \lambda)^{3}(b-a)^{3}}
\end{aligned}
$$

and

$$
\begin{aligned}
I_{3} & =\int_{0}^{1} t f^{\prime \prime \prime}[t(\lambda a+(1-\lambda) b)+(1-t)(\lambda b+(1-\lambda) a)] d t \\
& =\frac{f^{\prime \prime}(\lambda a+(1-\lambda) b)}{(1-2 \lambda)(b-a)}-\frac{f^{\prime}(\lambda a+(1-\lambda) b)}{(1-2 \lambda)^{2}(b-a)^{2}}+\frac{f^{\prime}(\lambda b+(1-\lambda) a)}{(1-2 \lambda)^{2}(b-a)^{2}}
\end{aligned}
$$

Hence, we get

$$
\begin{aligned}
I= & \int_{0}^{1} t(1-t)(2 t-1) f^{\prime \prime \prime}[t(\lambda a+(1-\lambda) b)+(1-t)(\lambda b+(1-\lambda) a)] d t \\
= & \frac{f^{\prime}(\lambda a+(1-\lambda) b)-f^{\prime}(\lambda b+(1-\lambda) a)}{(1-2 \lambda)^{2}(b-a)^{2}} \\
& -\frac{6[f(\lambda a+(1-\lambda) b)+f(\lambda b+(1-\lambda) a)]}{(1-2 \lambda)^{3}(b-a)^{3}}+\frac{12}{(1-2 \lambda)^{4}(b-a)^{4}} \int_{\lambda b+(1-\lambda) a}^{\lambda a+(1-\lambda) b} f(x) d x .
\end{aligned}
$$

This completes the proof.

Q.E.D.

Remark 2.2. If we take $\lambda=1$ or $\lambda=0$ in Lemma 2.1, then the identity (2.1) reduces the identity (1.2) which is proved in [4].

Now, we state the main results:

Theorem 2.3. Let $f:[a, b] \rightarrow \mathbb{R}$ be a three times differentiable mapping on $(a, b)$ with $a<b$. If 
$\left|f^{\prime \prime \prime}\right|^{q}$ is $s$-convex on $[a, b]$ for same fixed $s \in(0,1]$ and $q \geq 1$, then the following inequality holds:

$$
\begin{aligned}
& \mid \frac{(1-2 \lambda)^{2}(b-a)}{12}\left[f^{\prime}(\lambda a+(1-\lambda) b)-f^{\prime}(\lambda b+(1-\lambda) a)\right] \\
& \quad-\frac{(1-2 \lambda)}{2}[f(\lambda a+(1-\lambda) b)+f(\lambda b+(1-\lambda) a)]+\frac{1}{b-a} \int_{\lambda b+(1-\lambda) a}^{\lambda a+(1-\lambda) b} f(x) d x \mid \\
& \leq \quad \frac{(1-2 \lambda)^{4}(b-a)^{3}}{192}\left(\frac{2^{2-s}\left(6+s+2^{s+2} s\right)}{(s+2)(s+3)(s+4)}\right)^{\frac{1}{q}} \\
& \quad \times\left(\left|f^{\prime \prime \prime}(\lambda a+(1-\lambda) b)\right|^{q}+\left|f^{\prime \prime \prime}(\lambda b+(1-\lambda) a)\right|^{q}\right)^{\frac{1}{q}}
\end{aligned}
$$

where $\lambda \in[0,1] \backslash\left\{\frac{1}{2}\right\}$.

Proof. Using Lemma 2.1, s-convexity of $\left|f^{\prime \prime \prime}\right|^{q}$ and well-known Hölder's inequality, we obtain

$$
\begin{aligned}
& \mid \frac{(1-2 \lambda)^{2}(b-a)}{12}\left[f^{\prime}(\lambda a+(1-\lambda) b)-f^{\prime}(\lambda b+(1-\lambda) a)\right] \\
& -\frac{(1-2 \lambda)}{2}[f(\lambda a+(1-\lambda) b)+f(\lambda b+(1-\lambda) a)]+\frac{1}{b-a} \int_{\lambda b+(1-\lambda) a}^{\lambda a+(1-\lambda) b} f(x) d x \mid \\
\leq & \frac{(1-2 \lambda)^{4}(b-a)^{3}}{12} \int_{0}^{1} t(1-t)|2 t-1|\left|f^{\prime \prime \prime}[t(\lambda a+(1-\lambda) b)+(1-t)(\lambda b+(1-\lambda) a)]\right| d t \\
\leq & \frac{(1-2 \lambda)^{4}(b-a)^{3}}{12}\left(\int_{0}^{1} t(1-t)|2 t-1| d t\right)^{1-\frac{1}{q}} \\
& \times\left(\int_{0}^{1} t(1-t)|2 t-1|\left|f^{\prime \prime \prime}[t(\lambda a+(1-\lambda) b)+(1-t)(\lambda b+(1-\lambda) a)]\right|^{q} d t\right)^{\frac{1}{q}} \\
\leq & \frac{(1-2 \lambda)^{4}(b-a)^{3}}{12}\left(\frac{1}{16}\right)^{1-\frac{1}{q}}\left(\left|f^{\prime \prime \prime}(\lambda a+(1-\lambda) b)\right|^{q} \int_{0}^{1} t^{s+1}(1-t)|2 t-1| d t\right. \\
& \left.+\left|f^{\prime \prime \prime}(\lambda b+(1-\lambda) a)\right|^{q} \int_{0}^{\frac{1}{q}} t(1-t)^{s+1}|2 t-1| d t\right)^{\cdot}
\end{aligned}
$$




$$
\begin{aligned}
= & \frac{(1-2 \lambda)^{4}(b-a)^{3}}{12}\left(\frac{1}{16}\right)^{1-\frac{1}{q}} \\
& \times\left(\frac{6+s+2^{s+2} s}{(s+2)(s+3)(s+4)}\left[\left|f^{\prime \prime \prime}(\lambda a+(1-\lambda) b)\right|^{q}+\left|f^{\prime \prime \prime}(\lambda b+(1-\lambda) a)\right|^{q}\right]\right)^{\frac{1}{q}} \\
= & \frac{(1-2 \lambda)^{4}(b-a)^{3}}{192}\left(\frac{2^{2-s}\left(6+s+2^{s+2} s\right)}{(s+2)(s+3)(s+4)}\right)^{\frac{1}{q}} \\
& \times\left(\left|f^{\prime \prime \prime}(\lambda a+(1-\lambda) b)\right|^{q}+\left|f^{\prime \prime \prime}(\lambda b+(1-\lambda) a)\right|^{q}\right)^{\frac{1}{q}}
\end{aligned}
$$

The proof of Theorem 2.3 is comleted.

Q.E.D.

Remark 2.4. If we take $\lambda=1$ or $\lambda=0$ in Theorem 2.3, then the inequality (2.2) reduces the inequality (1.3) which is proved in [4].

Theorem 2.5. Let $f:[a, b] \rightarrow \mathbb{R}$ be a three times differentiable mapping on $(a, b)$ with $a<b$. If $\left|f^{\prime \prime \prime}\right|^{q}$ is $s$-convex on $[a, b]$ for same fixed $s \in(0,1]$ and $q>1$, then the following inequality holds:

$$
\begin{aligned}
& \mid \frac{(1-2 \lambda)^{2}(b-a)}{12}\left[f^{\prime}(\lambda a+(1-\lambda) b)-f^{\prime}(\lambda b+(1-\lambda) a)\right] \\
& \quad-\frac{(1-2 \lambda)}{2}[f(\lambda a+(1-\lambda) b)+f(\lambda b+(1-\lambda) a)]+\frac{1}{b-a} \int_{\lambda b+(1-\lambda) a}^{\lambda a+(1-\lambda) b} f(x) d x \mid \\
& \leq \quad \frac{(1-2 \lambda)^{4}(b-a)^{3}}{96}\left(\frac{1}{p+1}\right)^{\frac{1}{p}}\left(\frac{2^{1-s}\left(s 2^{s}+1\right)}{(s+1)(s+2)}\right)^{\frac{1}{q}} \\
& \quad \times\left(\left|f^{\prime \prime \prime}(\lambda a+(1-\lambda) b)\right|^{q}+\left|f^{\prime \prime \prime}(\lambda b+(1-\lambda) a)\right|^{q}\right)^{\frac{1}{q}}
\end{aligned}
$$

where $\lambda \in[0,1] \backslash\left\{\frac{1}{2}\right\}$ and $\frac{1}{p}+\frac{1}{q}=1$.

Proof. Using Lemma 2.1, s-convexity of $\left|f^{\prime \prime \prime}\right|^{q}$ and well-known Hölder's inequality, we obtain

$$
\begin{aligned}
& \mid \frac{(1-2 \lambda)^{2}(b-a)}{12}\left[f^{\prime}(\lambda a+(1-\lambda) b)-f^{\prime}(\lambda b+(1-\lambda) a)\right] \\
& -\frac{(1-2 \lambda)}{2}[f(\lambda a+(1-\lambda) b)+f(\lambda b+(1-\lambda) a)]+\frac{1}{b-a} \int_{\lambda b+(1-\lambda) a}^{\lambda a+(1-\lambda) b} f(x) d x \mid
\end{aligned}
$$




$$
\begin{gathered}
\leq \frac{(1-2 \lambda)^{4}(b-a)^{3}}{12} \int_{0}^{1} t(1-t)|2 t-1|\left|f^{\prime \prime \prime}[t(\lambda a+(1-\lambda) b)+(1-t)(\lambda b+(1-\lambda) a)]\right| d t \\
\leq \frac{(1-2 \lambda)^{4}(b-a)^{3}}{12}\left(\int_{0}^{1} t^{p}(1-t)^{p}|2 t-1| d t\right)^{\frac{1}{p}} \\
\quad \times\left(\int_{0}^{1}|2 t-1|\left|f^{\prime \prime \prime}[t(\lambda a+(1-\lambda) b)+(1-t)(\lambda b+(1-\lambda) a)]\right|^{q} d t\right)^{\frac{1}{q}} \\
\leq \frac{(1-2 \lambda)^{4}(b-a)^{3}}{12}\left(\frac{1}{2^{2 p+1}(p+1)}\right)^{\frac{1}{p}} \\
\times\left(\left|f^{\prime \prime \prime \prime}(\lambda a+(1-\lambda) b)^{q} \int_{0}^{1}\right| 2 t-\left.1\left|t^{s} d t+\right| f^{\prime \prime \prime}(\lambda b+(1-\lambda) a)\right|^{q} \int_{0}^{1}|2 t-1|(1-t)^{s} d t\right)^{\frac{1}{q}} \\
\leq \quad \frac{(1-2 \lambda)^{4}(b-a)^{3}}{12}\left(\frac{1}{2^{2 p+1}(p+1)}\right)^{\frac{1}{p}}\left(\frac{s 2^{s}+1}{2^{s}(s+1)(s+2)}\right)^{\frac{1}{q}} \\
\quad \times\left(\left|f^{\prime \prime \prime}(\lambda a+(1-\lambda) b)\right|^{q}+\left|f^{\prime \prime \prime}(\lambda b+(1-\lambda) a)\right|^{q}\right)^{\frac{1}{q}} \\
\leq \quad \frac{(1-2 \lambda)^{4}(b-a)^{3}}{96}\left(\frac{1}{p+1}\right)^{\frac{1}{p}}\left(\frac{2^{1-s}\left(s 2^{s}+1\right)}{(s+1)(s+2)}\right)^{\frac{1}{q}} \\
\times\left(\left.\left|f^{\prime \prime \prime}(\lambda a+(1-\lambda) b)^{q}+\right| f^{\prime \prime \prime}(\lambda b+(1-\lambda) a)\right|^{q}\right)^{\frac{1}{q}}
\end{gathered}
$$

which is the inequality $(2.3)$.

Q.E.D.

Remark 2.6. If we choose $\lambda=0$ or $\lambda=1$ in Theorem 2.5, then the inequality (2.3) reduces the inequality (1.4).

Theorem 2.7. Let $f:[a, b] \rightarrow \mathbb{R}$ be a three times differentiable mapping on $(a, b)$ with $a<b$. If $\left|f^{\prime \prime \prime}\right|^{q}$ is $s$-convex on $[a, b]$ for same fixed $s \in(0,1]$ and $q \geq 1$, then the following inequality holds:

$$
\begin{aligned}
& \mid \frac{(1-2 \lambda)^{2}(b-a)}{12}\left[f^{\prime}(\lambda a+(1-\lambda) b)-f^{\prime}(\lambda b+(1-\lambda) a)\right] \\
& -\frac{(1-2 \lambda)}{2}[f(\lambda a+(1-\lambda) b)+f(\lambda b+(1-\lambda) a)]+\frac{1}{b-a} \int_{\lambda b+(1-\lambda) a}^{\lambda a+(1-\lambda) b} f(x) d x \mid \\
\leq & \frac{(1-2 \lambda)^{4}(b-a)^{3}}{24}\left(\frac{1}{(p+1)(p+3)}\right)^{\frac{1}{p}}\left(\frac{2}{(s+2)(s+3)}\right)^{\frac{1}{q}} \\
& \times\left(\left|f^{\prime \prime \prime}(\lambda a+(1-\lambda) b)\right|^{q}+\left|f^{\prime \prime \prime}(\lambda b+(1-\lambda) a)\right|^{q}\right)^{\frac{1}{q}}
\end{aligned}
$$


where $\lambda \in[0,1] \backslash\left\{\frac{1}{2}\right\}$ and $\frac{1}{p}+\frac{1}{q}=1$.

Proof. Using Lemma 2.1, s-convexity of $\left|f^{\prime \prime \prime}\right|^{q}$ and well-known Hölder's inequality, we have

$$
\begin{aligned}
& \mid \frac{(1-2 \lambda)^{2}(b-a)}{12}\left[f^{\prime}(\lambda a+(1-\lambda) b)-f^{\prime}(\lambda b+(1-\lambda) a)\right] \\
& -\frac{(1-2 \lambda)}{2}[f(\lambda a+(1-\lambda) b)+f(\lambda b+(1-\lambda) a)]+\frac{1}{b-a} \int_{\lambda b+(1-\lambda) a}^{\lambda a+(1-\lambda) b} f(x) d x \\
& \leq \frac{(1-2 \lambda)^{4}(b-a)^{3}}{12} \int_{0}^{1} t(1-t)|2 t-1|\left|f^{\prime \prime \prime}[t(\lambda a+(1-\lambda) b)+(1-t)(\lambda b+(1-\lambda) a)]\right| d t \\
& \leq \frac{(1-2 \lambda)^{4}(b-a)^{3}}{12}\left(\int_{0}^{1} t(1-t)|2 t-1|^{p} d t\right)^{\frac{1}{p}} \\
& \times\left(\int_{0}^{1} t(1-t)\left|f^{\prime \prime \prime}[t(\lambda a+(1-\lambda) b)+(1-t)(\lambda b+(1-\lambda) a)]\right|^{q} d t\right)^{\frac{1}{q}} \\
& \leq \frac{(1-2 \lambda)^{4}(b-a)^{3}}{12}\left(\frac{1}{2(p+1)(p+3)}\right)^{\frac{1}{p}} \\
& \times\left(\left|f^{\prime \prime \prime}(\lambda a+(1-\lambda) b)\right|^{q} \int_{0}^{1} t^{s+1}(1-t) d t+\left|f^{\prime \prime \prime}(\lambda b+(1-\lambda) a)\right|^{q} \int_{0}^{1} t(1-t)^{s+1} d t\right)^{\frac{1}{q}} \\
& =\frac{(1-2 \lambda)^{4}(b-a)^{3}}{12}\left(\frac{1}{2(p+1)(p+3)}\right)^{\frac{1}{p}}\left(\frac{1}{(s+2)(s+3)}\right)^{\frac{1}{q}} \\
& \times\left(\left|f^{\prime \prime \prime}(\lambda a+(1-\lambda) b)\right|^{q}+\left|f^{\prime \prime \prime}(\lambda b+(1-\lambda) a)\right|^{q}\right)^{\frac{1}{q}} \\
& =\frac{(1-2 \lambda)^{4}(b-a)^{3}}{24}\left(\frac{1}{(p+1)(p+3)}\right)^{\frac{1}{p}}\left(\frac{2}{(s+2)(s+3)}\right)^{\frac{1}{q}} \\
& \times\left(\left|f^{\prime \prime \prime}(\lambda a+(1-\lambda) b)\right|^{q}+\left|f^{\prime \prime \prime}(\lambda b+(1-\lambda) a)\right|^{q}\right)^{\frac{1}{q}} .
\end{aligned}
$$

Q.E.D.

Remark 2.8. If we take $\lambda=0$ or $\lambda=1$ in Theorem 2.7, then the inequality (2.4) becomes the inequality (1.5). 


\section{References}

[1] A. G. Azpeitia, Convex functions and the Hadamard inequality, Rev. Colombiana Math., 28 (1994), 7-12.

[2] M. K. Bakula and J. Pečarić, Note on some Hadamard-type inequalities, Journal of Inequalities in Pure and Applied Mathematics, vol. 5, no. 3, article 74, 2004.

[3] W. W. Breckner, Stetigkeitsaussagen für eine Klasse verallgemeinerter konvexer funktionen in topologischen linearen Raumen, Pupl. Inst. Math. 23(1978), 13-20.

[4] L. Chun and F. Qi, Integral Inequalities of Hermite-Hadamard Type for Functions Whose 3rd Derivatives Are s-Convex, Applied Mathematics, 2012, 3, 1680 1685.http://dx.doi.org/10.4236/am.2012.311232.

[5] L. Chun and F. Qi, Integral inequalities of Hermite-Hadamard type for functions whose third derivatives are convex, Journal of Inequalities and Applications 2013, 2013:451 doi:10.1186/1029-242X-2013-451.

[6] S. S. Dragomir and C. E. M. Pearce, Selected Topics on Hermite-Hadamard Inequalities and Applications, RGMIA Monographs, Victoria University, 2000.

[7] S. S. Dragomir and R.P. Agarwal, Two inequalities for differentiable mappings and applications to special means of real numbers and to trapezoidal formula, Appl. Math. lett., 11(5) (1998), 91-95.

[8] H. Hudzik and L. Maligranda, Some remarks on $s$-convex functions, Aequationes Math. 48 (1994), 100-111.

[9] J. Park, Hermite-Hadamard Type Inequalities for Functions whose Third Derivatives are Convex and s-Convex, Applied Mathematical Sciences, Vol. 8, 2014, no. 1, 13 - 31

[10] J. E. Pečarić, F. Proschan and Y. L. Tong, Convex Functions, Partial Orderings and Statistical Applications, Academic Press, Boston, 1992.

[11] S. Qaisar, S. Hussain and C. He, On new inequalities of Hermite-Hadamard type for functions whose third derivative absolute values are quasi-convex with applications, Journal of the Egyptian Mathematical Society (2014) 22, 19-22.

[12] B-Y. Xi, S-H. Wang and F. Qi, Some Inequalities of Hermite-Hadamard Type for Functions Whose 3rd Derivatives Are P-Convex, Applied Mathematics, 2012, 3, 1898-1902. 